\title{
Prevalence of attention-deficit/ hyperactivity disorder (ADHD) symptomatology and psychiatric co-morbidity among adolescents diagnosed with ADHD in childhood
}

S Walker, MSocSc, Couns Psych, PhD

Unit for Professional Training and Service in the Behavioural Sciences, University of the Free State, Bloemfontein

\section{A Venter, MMed (Paed), PhD}

Department of Paediatrics and Child Health, University of the Free State

\section{A van der Walt, MMed (Paed)}

Private Practice, Panorama Hospital, and Part-time Consultant in the Department of Paediatrics and Child Health, Red Cross Hospital

K G F Esterhuyse, PhD

Department of Psychology, University of the Free State

Objectives. Given the paucity of research on adolescent attention-deficit/hyperactivity disorder (ADHD), this study aimed to establish the prevalence of Diagnostic and Statistical Manual of Mental Disorders, 4th edition (DSM-IV) ADHD in a cohort of South African adolescents who had been diagnosed with the disorder in childhood. It also aimed to establish the prevalence of psychiatric co-morbidities and adjustment difficulties in this sample.

Method. Data regarding age of diagnosis, current ADHD status, current ADHD-related pharmacological management, current psychopathology and current adjustment were gathered from 64 adolescents and their guardians via self-report questionnaire. Descriptive statistics were calculated with regard to current ADHD status, co-morbid psychopathology and adjustment difficulties, as well as current ADHD-related medication.

Results. According to parent reports, 59.38\% of the sample met DSM-IV criteria for ADHD Inattentive subtype, while $37.50 \%$ met the criteria for ADHD Hyperactive/Impulsive subtype. Of the adolescents, $64.06 \%$ were still using stimulant medication. Based on the adolescent self-report, $43.75 \%$ of the sample had clinically significant symptoms of psychopathology or maladjustment. Furthermore, $39.28 \%$ met the diagnostic criteria for at least one psychiatric co-morbidity.

Conclusion. ADHD persisted into adolescence in the current sample. A significant psychopathological and maladjustment load appears evident among adolescents previously diagnosed with ADHD, despite continuous pharmacological management of the condition.

It was once thought that attention-deficit/hyperactivity disorder (ADHD) did not persist into adolescence, but results from two prospective studies suggest otherwise. ${ }^{13}$ The results of a meta-analysis suggest a $15 \%$ persistence rate of ADHD into adolescence when the full Diagnostic and Statistical Manual of Mental Disorders, 4th edition (DSM-IV) diagnostic criteria are used. ${ }^{3}$ However, it has been postulated that persistence rates of ADHD into adolescence would be much higher if adolescents meeting the partial remission criteria were also included. ${ }^{4}$ With this in mind, a recent study of 157 individuals who had been diagnosed with ADHD between the ages of 6 and 15 years found that on the basis of parents' reports, $60 \%$ of these individuals still met the DSM-IV criteria for ADHD 5 years after diagnosis. ${ }^{5}$ There would therefore appear to be convincing evidence of noteworthy persistence rates into adolescence for both full DSM-IV criteria and partial-remission ADHD.

Longitudinal studies of children with ADHD indicate that the majority of individuals diagnosed with the disorder during childhood continue to exhibit clinically significant symptom levels during adolescence. A recent study found that in a sample of 296 individuals diagnosed with DSM-IV ADHD at a mean age of 6 years, $68.8 \%$ still had persistent ADHD between the ages of 11 and 17 years. Most adolescents suffering from ADHD report higher levels of emotional, social and scholastic impairment than their non-ADHD peers.' Moreover, psychiatric co-morbidities appear to be the norm rather than the exception among adolescents diagnosed with ADHD. ${ }^{8}$ The most commonly occurring co-morbid conditions are reported to be oppositional defiant disorder (ODD), conduct disorder (CD), mood disorders, anxiety disorders, learning disorders and substance abuse disorders. Co-morbid ODD and/or CD have been reported in 
$30-45 \%$ of children diagnosed with ADHD by the time they reached 15 years of age. Anxiety was evident in $30 \%$ of the same sample, while rates of depression and bipolar mood disorder were reported to be $45 \%$ and $23 \%$, respectively. Youth with persistent ADHD have been found to be at higher risk for anxiety disorders than controls and more likely to meet the DSMIV diagnostic criteria for ODD than youth with partially remitted ADHD. ${ }^{\circ}$ However, both individuals suffering from persistent ADHD and those with partially remitted ADHD have been found to be significantly more likely than controls to be diagnosed with $O D D$, $C D$, tics, mood disorders, past and regular use of substances, and sleep disorders. ${ }^{5}$ It would therefore appear that while persistent ADHD places adolescents at increased risk for a variety of psychopathologies, reduced ADHD symptoms in adolescents previously diagnosed with the disorder do not necessarily imply reduced risk for psychiatric co-morbidities.

It appears evident from the literature that $A D H D$ continues into adolescence. Moreover, adolescents suffering from ADHD seem to be at increased risk of developing psychiatric co-morbidities and exhibit lower levels of scholastic functioning and lower levels of adjustment than adolescents without a history of ADHD. However, ADHD in adolescence has received relatively little attention in the international research literature and practically none locally. This study therefore aimed to establish the prevalence of DSM-IV ADHD in a cohort of South African adolescents who had been diagnosed with ADHD in childhood, as well as the prevalence of current co-morbidities.

\section{Method \\ Participants}

Two paediatric neurodevelopmentalists in private practice in the Free State and Western Cape agreed to review their practice files in order to identify adolescents who had previously been diagnosed with ADHD. These practitioners then contacted the guardians/parents of the adolescents concerned to obtain consent for the researchers to approach the guardians and adolescents regarding participation in the study. This procedure yielded a pool of 300 adolescents previously diagnosed with ADHD who consented to be approached. Letters detailing the aims of the study were mailed to the adolescents' guardians/ parents along with informed consent forms and questionnaires for both the guardians/parents and the adolescents to complete. All guardians/parents were provided with self-addressed envelopes and were requested to mail their completed questionnaires, as well as the questionnaire completed by the adolescent participants, back to the researchers. The adolescents were requested to seal their completed questionnaires in envelopes provided by the researchers before handing them to their guardians/parents so as to ensure confidentiality. This methodology yielded 64 complete data sets (21.33\% response rate). Minimum response rates of $20 \%$ are recommended for mail-based survey research. ${ }^{10}$

\section{Measures}

Adolescent participants completed the Adolescent Psychopathology Scale - Short Form (APS-SF)." The APS-SF is a brief self-report measure of symptoms of psychopathology and emotional distress in adolescents. The measure was designed to be consistent with DSM-IV symptom specification and yields scores on 12 clinical scales and 2 validity scales. Reliability coefficients of between 0.80 (Conduct Disorder and Self-Concept) and 0.91 (Major Depression) are reported for the clinical scales, while reliability coefficients for the validity scales range between 0.46 for the Defensiveness scale to 0.48 for the Consistency Response scale.

The guardians/parents of the adolescents previously diagnosed with ADHD completed the Swanson, Nolan and Pelham ISNAPIV) rating scale based on their observations of the adolescents. ${ }^{12}$ Guardians/parents also completed a biographical questionnaire relating to the adolescent's age at diagnosis, scholastic history, current medications and other pertinent information.

\section{Results}

As stated previously, 64 completed sets /guardians/parents and adolescents) of questionnaires were received. Scores for the SNAP-IV ADHD Inattentive, Hyperactive/Impulsive and Combined subscales were calculated independently. Adolescent participants' responses to the APS-SF were scored using the Adolescent Psychopathology Scale - Short Form Scoring Program for Windows. ${ }^{13}$ Only APS-FS scores elevated into the moderate clinical symptom range or severe clinical symptom range were included in the analyses. This was done in an attempt to raise the diagnostic threshold and improve specificity. All data were encoded and descriptive statistics pertaining to demographic variables, current ADHD status, current psychopathology symptomatology and adjustment difficulties, and current medications were calculated using SPSS computer software."

The data in Table I provide a general overview of the demographic characteristics of the sample. The sample was predominantly male (81.25\%). Approximately half of the participants were resident in the Western Cape (53.13\%) with the Free State and Gauteng being home to $23.44 \%$ and $12.50 \%$, respectively. Most participants appeared to come from socio-economically advantaged backgrounds, with $59.38 \%$ of the guardians/parents reporting a personal monthly income of R15 000 or more. Despite nearly half the sample (48.44\%) reporting having received a learning disability diagnosis of some form, $62.90 \%$ reported never having repeated a grade at school and only $12.91 \%$ claimed to have repeated a grade more than once. The average age at which the adolescents were reported to have been diagnosed with ADHD was 8.03 years (standard deviation (SD) 2.986 years). The average age of the participants at the time of the study was 15.66 years (SD 1.428 years). 


\begin{tabular}{|c|c|c|}
\hline & Frequency & $\%$ \\
\hline \multicolumn{3}{|l|}{ Gender $(N=64)$} \\
\hline Male & 52 & 81.25 \\
\hline Female & 12 & 18.75 \\
\hline \multicolumn{3}{|l|}{$\begin{array}{l}\text { Geographical distribution of the } \\
\text { sample }(N=64)\end{array}$} \\
\hline Eastern Cape & 2 & 3.13 \\
\hline Free State & 15 & 23.44 \\
\hline Gauteng & 8 & 12.5 \\
\hline North West & 1 & 1.56 \\
\hline Northern Cape & 4 & 6.25 \\
\hline Western Cape & 34 & 53.13 \\
\hline \multicolumn{3}{|l|}{$\begin{array}{l}\text { Adult informant's monthly } \\
\text { income }(N=64)\end{array}$} \\
\hline$\leq R 1500$ & 3 & 4.69 \\
\hline R1 $501-3000$ & 3 & 4.69 \\
\hline R3 $001-5500$ & 2 & 3.13 \\
\hline R5 501 - 8000 & 3 & 4.69 \\
\hline R8 $001-10500$ & 4 & 6.25 \\
\hline R10 $501-15000$ & 10 & 15.63 \\
\hline$\geq \mathrm{R} 15000$ & 38 & 59.38 \\
\hline No response & 1 & 1.56 \\
\hline \multicolumn{3}{|l|}{ No. of grades repeated $(N=63)$} \\
\hline 0 & 39 & 62.90 \\
\hline 1 & 15 & 24.19 \\
\hline 2 & 6 & 9.68 \\
\hline 3 & 2 & 3.23 \\
\hline Learning disability diagnoses $(N=64)$ & 31 & 48.44 \\
\hline
\end{tabular}

Table II reflects the frequencies of ADHD subtypes currently evident in the adolescent respondents based on their guardians' / parents' responses on the SNAP-IV, as well as the adolescents' current

\begin{tabular}{lcc}
$\begin{array}{l}\text { Table II. Frequency distribution for ADHD subtypes and } \\
\text { psychiatric co-morbidities }\end{array}$ & Frequency & $\%$ \\
\hline & 38 & 59.38 \\
\hline & 24 & 37.50 \\
ADHD subtype (N=64)* & 25 & 39.07 \\
Inattentive & & \\
Hyperactive/Impulsive & 6 & 21.43 \\
Combined & 5 & 17.86 \\
Psychiatric co-morbidities (N=28) & 3 & 10.71 \\
Conduct disorder & 8 & 28.57 \\
Oppositional defiant disorder & 8 & 28.57 \\
Substance abuse disorder & 5 & 17.86 \\
Generalised anxiety disorder & 13 & 46.43 \\
Post-traumatic stress disorder & 9 & 32.14 \\
Major depression & 7 & 25.00 \\
Eating disturbance & 8 & 28.57 \\
Suicide proneness & 8 & 28.57 \\
Poor self-concept & 10 & 35.71 \\
Anger/violence proneness & & \\
Academic problems & \multicolumn{3}{l}{} \\
Interpersonal problems &
\end{tabular}

psychopathological and adjustment status, based on their own responses on the APS-SF. According to their guardians' / parents' responses on the SNAP-IV, 59.38\% of the sample met the DSM-IV criteria for ADHD (Inattentive subtype). Twenty-four participants (37.50\%) appeared to meet the criteria for ADHD (Hyperactive/ Impulsive subtype), while 25 participants (39.07\%) met the criteria for the combined subtype. Significant symptom loading in all three subtypes of ADHD was calculated in order to obtain an impression of the behavioural profile of the participants' $A D H D$ symptoms. Consequently, participants were not categorised according to specific diagnoses. The result is that the same individual may be represented in the Hyperactive/Impulsive category or the Impulsive category, as well as being assigned to the Combined category. However, regardless of the specific approach to categorisation, it appears evident that the majority of the adolescents still met the criteria for ADHD (Inattentive subtype) and that a significant proportion meet the criteria for ADHD (Hyperactive/Inattentive subtype). These findings suggest that $\mathrm{ADHD}$ symptomatology continues to be apparent throughout adolescence.

It is evident from Table II that $43.75 \%$ of the adolescent participants reported suffering from clinically significant symptoms of psychopathology or maladjustment. Of the 28 individuals reporting some form of psychopathology or maladjustment, $39.28 \%$ (11 individuals) appeared to meet the criteria for only one psychiatric/adjustment diagnosis, while a further $21.43 \%$ (6 individuals) seemed to meet the criteria for two diagnoses and $12(42.28 \%)$ to meet the criteria for three or more diagnoses. There would therefore appear to be noteworthy co-morbid psychopathology and adjustment difficulties associated with adolescent ADHD. It is apparent from Table $\|$ that eating disturbances, difficulties in social functioning, problems in close interpersonal relationships and behaviour regulation difficulties, as well as suicidal ideation and thoughts related to self-harm, were the forms of psychopathology/maladjustment most frequently reported by the sample. The reported eating disturbances may be related to loss of appetite as a side-effect of stimulant medication rather than indicate an eating disorder per se. Elevated frequencies of academic difficulties, anger, poor self-concept, conduct problems, generalised anxiety and posttraumatic stress are also noted in Table II. It should be noted that items on the APS-SF that comprise the Generalised Anxiety and Post-traumatic Stress scales sample irritability, restlessness, sleeprelated difficulties and concentration problems along with the core anxiety-related features of these disorders. It is therefore possible that the elevated levels of generalised anxiety and post-traumatic symptomatology among the participants could be more accurately ascribed to clinically significant levels of inattention, irritability and restlessness than to the presence of generalised anxiety disorder (GAD) or post-traumatic stress disorder (PTSD) as such.

Table III shows that only $23.44 \%$ of the adolescents participating in the study reported not using any prescription medication. 


\begin{tabular}{lcc} 
Table III. Current medications $(\mathbf{N}=\mathbf{6 4})$ & & \\
\hline Medication & Frequency & $\%$ \\
\hline None & 15 & 23.44 \\
Ritalin & 21 & 32.81 \\
Concerta & 20 & 31.25 \\
Strattera & 2 & 3.13 \\
Risperdal & 4 & 6.25 \\
Anticonvulsant & 1 & 1.56 \\
Tricyclic antidepressant & 2 & 3.13 \\
SSRI & 4 & 6.25 \\
Allergy medication & 5 & 7.81 \\
Other & 3 & 4.69 \\
\hline
\end{tabular}

Approximately two-thirds (64.06\%) of the sample reported using stimulant medication, while a further $6.25 \%$ of the participants report using Risperdal and a similar percentage report using selective serotonin reuptake inhibitors (SSRIs). It would appear that a significant number of the adolescents in the sample continued to be actively medicated for ADHD. Nine participants 1 14.06\% of the total sample) were reported to be on dual therapy. Of these, most were reported to be on combinations of either a stimulant and an SSRI or a stimulant and a neuroleptic.

\section{Discussion}

The primary aim of this study was to gain a deeper understanding of the prevalence of ADHD symptomatology among South African adolescents who had been diagnosed with ADHD as children. The study also aimed to determine the extent to which these adolescents currently met the DSM-IV diagnostic criteria for $A D H D$, as well as the prevalence of psychiatric disorders and adjustment difficulties in this group.

Parents'/guardians' responses to the SNAP-IV revealed that $59.38 \%$ of the adolescents in the study met the DSM-IV criteria for ADHD Inattentive subtype, while $37.50 \%$ and $39.07 \%$ met the criteria for ADHD Hyperactive/Impulsive and Combined subtypes, respectively. It is important to note that as the study aimed to determine the prevalence of ADHD symptomatology and not subtypes of $A D H D$, criteria for each of the ADHD subtypes were assessed separately. Consequently, participants who met the criteria for ADHD Inattentive or Hyperactive/ Impulsive subtypes may also be included among those meeting the criteria for ADHD Combined subtype. Nonetheless, these findings suggest that the majority of individuals in this sample who were diagnosed with $A D H D$ as children continue to display significant $\mathrm{ADHD}$ symptomatology during adolescence. It would therefore appear that the persistence rates of childhood ADHD into adolescence reported in this study are comparable to those reported in other countries. ${ }^{5,6}$ The higher prevalence of primarily inattentive symptoms is in keeping with trends in other studies, as is the general assumption that while hyperactive and impulsive behaviour becomes less prominent as individuals with ADHD age, symptoms of inattention remain prevalent. ${ }^{0.7}$

This particular sample of adolescents reported a significant number of psychiatric and adjustment difficulties. Almost half $(43.75 \%)$ of the sample's self-reports reflected clinically significant symptoms of psychopathology or maladjustment. Moreover, of the 28 individuals meeting the criteria for psychiatric co-morbidities and adjustment problems, 12 (42.28\%) appeared to meet the criteria for three or more co-morbidities. Eating-related difficulties, interpersonal problems, poor behaviour regulation and thoughts of self-harm appear to be the difficulties most frequently reported by the sample. With the exception of eating-related difficulties, which could possibly be ascribed to the side-effects of stimulant medication, the most frequently reported problems appear to involve the adolescents' ability to relate effectively to their social environment, as well as their apparent inability to deal effectively with frustration. It could therefore be hypothesised that in addition to being at higher risk for psychopathology, individuals with ADHD appear to be less capable of dealing effectively with the developmental challenges of adolescence. Consequently, they may be more inclined to present with exaggerated or extreme forms of behavioural and emotional difficulties commonly associated with normal adolescent development. It should also be noted that high rates of learning disability diagnoses (48.44\%) are reported in the current sample. This is very much in keeping with international literature supporting a link between ADHD and learning difficulties. ${ }^{7.15}$

It has been stated previously that some degree of symptom overlap may be evident in the diagnostic categories yielded by the APS-SF. Consequently, certain diagnoses may be overrepresented, e.g. PTSD and GAD. However, this should not detract from the high frequency of clinically relevant symptoms of psychopathology and maladjustment reported by the participants. Stated differently, even if these individuals do not present with the number of psychiatric conditions suggested by the APS-SF, they still report a high rate of emotional distress, disruptive behaviour and functional impairment. It is thus evident that individuals in this sample who have been diagnosed with ADHD during childhood present with increased rates of psychiatric co-morbidity and maladjustment during adolescence. Moreover, these findings are comparable to rates of psychiatric co-morbidities reported for samples of adolescent ADHD sufferers in other countries.

The findings of the current study would suggest that significant ADHD symptomatology persists into adolescence and that significant rates of psychopathology and adjustment difficulties can be associated with adolescent ADHD. These findings are of particular interest given the demographics of the sample. The adolescents in this sample had on average been diagnosed with ADHD at the age of 8 years, and $64.06 \%$ were reported still to be using stimulant medication at the time of data collection. Furthermore, $59.38 \%$ of the adolescents' parents reported 
a personal income of R15000 or more per month. It would therefore appear that the current sample consists predominantly of individuals from higher socio-economic backgrounds who were diagnosed with ADHD during childhood and appear not only to have been optimally treated for the condition but still to be on medication. Such individuals could be expected to experience better outcomes overall. Consequently, it could be assumed that although the persistence rates and co-morbidities in this group appear high, they are probably lower than those in individuals with less favourable social and medical histories.

This study is not without certain methodological limitations. Firstly, the sample appears fairly homogeneous and it is doubtful whether these findings could be generalised to adolescent ADHD sufferers from very different backgrounds. More specifically, adolescents in the current sample generally appeared to come from socioeconomically advantaged backgrounds. As a result it may be assumed that they have had access to treatment resources and other forms of assistance (remedial education interventions, behaviour management interventions, etc.) that might not be available to the majority of adolescents suffering from ADHD. The current sample would therefore seem to comprise adolescents with comparatively favourable prognoses. Secondly, the purposive sampling method we followed may also have skewed the final sample with regard to diagnosis and management. The high rates of $\mathrm{ADHD}$ symptomatology and continued medication use reported by the participants may suggest that individuals and families still significantly affected by ADHD were perhaps more inclined to participate in the study than those less affected. Consequently, the sample may be overly representative of persistent ADHD and associated co-morbidities. In all probability the sampling method resulted in more skewed findings than would have been the case in a more broad-based epidemiological study making use of more representative sampling methods. Furthermore, future research would do well to adopt a longitudinal approach to studying the developmental course of adjustment difficulties and psychiatric co-morbidities related to ADHD through childhood into adolescence. Thirdly, the total reliance on self-report measures and parent report may have resulted in less reliable information than direct clinical observation and teacher ratings.

\section{Conclusion}

The majority of cases of ADHD diagnosed in childhood seem to persist into adolescence. Adolescents who were diagnosed with ADHD during childhood appear to be at particular risk for co-morbid psychopathology, adjustment problems and learning difficulties. These trends are evident despite initial treatment of the condition and continued medication use during adolescence.

\section{References}

1. Biederman J, Monuteaux MC, Mick E, et al. Psychopathology in females with attention-deficit/hyperactivity disorder: a controlled, five-year prospective study. Biol Psychiatry 2006;60:1098-1 110 .

2. Biederman J. Attention-deficit/hyperactivity disorder: a selective overview. Biol Psychiatry 2005;57:1215-1220

3. Faraone SV, Biederman J, Mick E. The age-dependent decline of attention deficit hyperactivity disorder: a meta-analysis of follow-up studies. Psychol Med 2006; 36:159-165.

4. Sciberras $E$, Roos $L E$, Efron D. Review of prospective longitudinal studies of children with ADHD: mental health, educational, and social outcomes. Current Attention Disorders Reports 2009;1:171-177

5. Ford T, Fowler T, Langley K, Whittinger $N$, Thapar A. Five years on: public sector service use related to mental health in young people with ADHD or hyperkinetic disorder five years after diagnosis. Child Adolesc Ment Health 2008;1 3:1 122-1 29.

6. Gau SS, Ni HC, Shang CY, et al. Psychiatric comorbidities among children and adolescents with and without persistent attention-deficit/hyperactivity disorder. Aust N ZJ Psychiatry 2010;44:135-143

7. Lee SS, Lahey BB, Owens EB, Hinshaw SP. Few preschool boys and girls with ADHD are well-adjusted during adolescence. J Abnorm Child Psychol 2008;36:373-383.

8. Schubiner $\mathrm{H}$, Katragadda S. Overview of epidemiology, clinical features, genetics, neurobiology, and prognosis of adolescent attention-deficit/hyperactivity disorder. Adolesc Med State Art Rev 2008; 19;209-215.

9. Biederman J, Newcorn J, Sprich, S. Comorbidity of attention deficit hyperactivity disorder with conduct, depressive, anxiety, and other disorders. Am J Psychiatry $1991 ; 148: 564-577$

10. Babbie ER. The Practice of Social Research. 7th ed. Belmont: Wadsworth, 1995.

11. Reynolds WM. Adolescent Psychopathology Scale-Short Form. Lutz: Psychological Assessment Resources Inc, 2000.

12. Swanson JM, Kraemer HC, Hinshaw SP, et al. Clinical relevance of the primary findings of the MTA: Success rates based on severity of ADHD and ODD symptoms at the end of treatment. J Am Acad Child Adolesc Psychiatry 200 1;40: 168-179.

13. Reynolds WM. Adolescent Psychopathology Scale-Short Form Scoring Program Version 1.00.007. Lutz: Psychological Assessment Resources Inc, 2004.

14. SPSS Incorporated. SPSS User's Guide: Version 12.0. New York: SPSS Incorporated, 2001

15. Molina BSG, Pelham WE. Substance use, substance abuse and LD among adolescents with a childhood history of ADHD. J Learn Disabil 2001;34:333-342. 